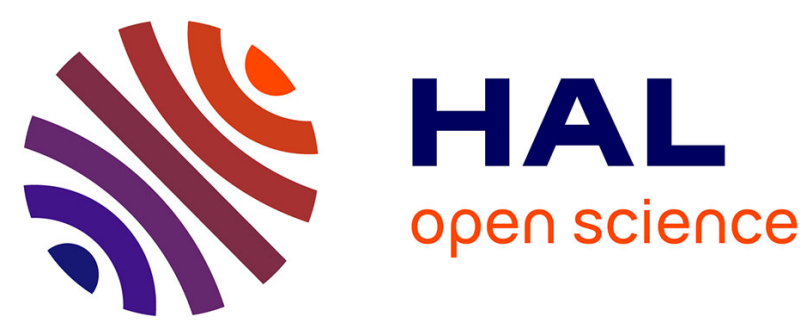

\title{
Coherent combining of difference frequency generators: experimental demonstration and application to optical parametric oscillators
}

Pierre Bourdon, Rodwane Chtouki, Anne Durecu, Laurent Lombard, Christophe Planchat, Myriam Raybaut, Antoine Godard

\section{To cite this version:}

Pierre Bourdon, Rodwane Chtouki, Anne Durecu, Laurent Lombard, Christophe Planchat, et al.. Coherent combining of difference frequency generators: experimental demonstration and application to optical parametric oscillators. SPIE Photonics West 2019, Feb 2019, SAN FRANCISCO, United States. 10.1117/12.2509933 . hal-02127017

\section{HAL Id: hal-02127017 https://hal.science/hal-02127017}

Submitted on 13 May 2019

HAL is a multi-disciplinary open access archive for the deposit and dissemination of scientific research documents, whether they are published or not. The documents may come from teaching and research institutions in France or abroad, or from public or private research centers.
L'archive ouverte pluridisciplinaire HAL, est destinée au dépôt et à la diffusion de documents scientifiques de niveau recherche, publiés ou non, émanant des établissements d'enseignement et de recherche français ou étrangers, des laboratoires publics ou privés. 


\title{
Coherent combining of difference frequency generators: experimental demonstration and application to optical parametric oscillators
}

\author{
P. Bourdon ${ }^{*}$, R. Chtouki, A. Durécu, L. Lombard, C. Planchat, M. Raybaut, A. Godard \\ ONERA, The French Aerospace Lab, BP 80100, 91123 Palaiseau Cedex, France
}

\begin{abstract}
Coherent beam combining (CBC) by active phase control could be useful for power scaling fiber-laser-pumped optical frequency converters like optical parametric oscillators (OPOs). We developed an indirect phase control approach based on the phase matching relation intrinsic to efficient nonlinear processes.

Previously, we demonstrated coherent combining of second harmonic waves through real time active control of the phases of the fundamental waves, using high bandwidth fibered electro-optic phase modulators. In the case of this 2 wavelength process, it was possible to simultaneously combine both the fundamental and the second harmonic waves.

In this paper, we present an experimental demonstration of coherent combining of difference frequency generators emitting an idler wave at $3400 \mathrm{~nm}$. We confirm experimentally the theoretical prediction that through active phase control of the sole $1064 \mathrm{~nm}$ pump waves, it's possible to coherently combine the idler waves efficiently. A residual phase error of $1 / 28$ th wave at $3400 \mathrm{~nm}$ is achieved, corresponding to an excellent combining efficiency.

However, in such a 3-wavelength process, simultaneous combination of the signal and idler waves is not always feasible.

This demonstration opens the way to mid-infrared OPO combining. We present the architectures of continuous wave OPOs we are working on.
\end{abstract}

Keywords: coherent beam combining, nonlinear optics, difference frequency generation, optical parametric oscillator

\section{INTRODUCTION}

Coherent beam combining (CBC) through active phase control is a common solution for power scaling fiber lasers to overcome power limitations induced by nonlinear effects such as stimulated Brillouin or Raman scattering. But nonlinear frequency converters such as parametric generators used in gas sensing could also benefit a lot from CBC power scaling, as it would improve the detection range or the sensitivity of the instrument.

For instance, most often, nonlinear crystals are limited in aperture due to the technological and financial cost of growing large crystals, or for periodically poled crystals, due to technical limitations of the poling process. Such crystals with limited apertures have to be pumped using narrow beams. Hence, the level of power generated has to be kept below the damage threshold of the nonlinear material. Power scaling capability offered by CBC could be useful to overcome these limitations.

We demonstrated previously ${ }^{1}$ the feasibility of indirect phase control for second harmonic generators combination, taking advantage of the fact that nonlinear frequency conversion is a phase sensitive process which is strongly dependant on the relative phases of the interacting waves. This phase relation was used to indirectly control the phase of the harmonic waves through controlling the phases of the fundamental waves. With this indirect phase control technique, we are also able to use fast standard all-fiber $1-\mu \mathrm{m}$ or $1.5-\mu \mathrm{m}$ wavelength electro-optic phase modulator (EOM), very compact and efficient components that are not easily available at higher wavelengths.

*pierre.bourdon@onera.fr; phone (+33)-1-80-38-63-82; fax(+33)-1-80-38-63-45; www.onera.fr 
In $2017^{2}$, we proposed a scheme of experiment to demonstrate that indirect phase control can only operate to combine difference frequency generators (DFG) in the mid-infrared. Since then, this experiment has been performed and we present here the experimental results obtained and the demonstration of coherent combining of DFG modules operating at $3.4 \mu \mathrm{m}$ by active phase control of their $1.064-\mu \mathrm{m}$ pump waves.

\section{PRINCIPLE OF COHERENT COMBINING OF DFG CONVERTERS}

The theoretical principle behind the capability for indirect phase control of DFG waves is easy to demonstrate solving the classical nonlinear optics propagation equations. We use the following notations to express the electric field for the pump wave $\left(\omega_{1}\right)$, the idler wave $\left(\omega_{2}\right)$ and the signal wave $\left(\omega_{3}\right)$. To simplify this explanation, we assume the beams to be linearly polarized, and use scalar and not vector expressions and equations.

The electric field corresponding to each nonlinearly coupled wave can be written as:

$$
\widetilde{E}_{m}(z, t)=A_{m}(z) e^{j k_{m} z} e^{-j \omega_{m} t}+c . c
$$

where $A_{j}$ is the complex amplitude of the $\omega_{j}$ wave.

If we assume that the pump wave is not depleted when passing through the crystal, the coupled-amplitude equations describing the difference frequency generation are:

$$
\left\{\begin{array}{l}
\frac{d A_{\mathrm{s}}(\mathrm{z})}{d z}=\frac{8 \pi j \omega_{\mathrm{s}}^{2} d_{e f f}}{k_{\mathrm{s}} c^{2}} A_{\mathrm{p}} A_{\mathrm{i}}^{*}(z) e^{j \Delta k z} \\
\frac{d A_{\mathrm{i}}(z)}{d z}=\frac{8 \pi j \omega_{\mathrm{i}}^{2} d_{e f f}}{k_{\mathrm{i}} c^{2}} A_{\mathrm{p}} A_{\mathrm{s}}^{*}(z) e^{j \Delta k z}
\end{array}\right.
$$

where $\Delta k=k_{p}-k_{S}-k_{i}$.

If we assume that the idler wave is not seeded, we can consider that $A_{i}(0)=0$. The initial value for the signal wave amplitude is noted $A_{s}(0)$. Thus, equations (2) can be solved using the notation $\kappa_{\mathrm{m}}=\frac{8 \pi \mathrm{j} \omega_{\mathrm{m}}^{2} \mathrm{~d}_{\mathrm{eff}}}{\mathrm{k}_{\mathrm{m}} \mathrm{c}^{2}}$ :

$$
\left\{\begin{array}{c}
A_{\mathrm{s}}(\mathrm{z})=A_{\mathrm{s}}(0) \cosh \left(\sqrt{\kappa_{\mathrm{s}} \kappa_{\mathrm{i}}^{*}\left|A_{\mathrm{p}}\right|^{2}} \mathrm{z}\right) \\
A_{\mathrm{i}}(\mathrm{z})=\frac{\kappa_{\mathrm{i}} A_{\mathrm{p}}(0)}{\sqrt{\kappa_{\mathrm{s}} \kappa_{\mathrm{i}}^{*}\left|A_{\mathrm{p}}\right|^{2}}} A_{\mathrm{s}}^{*}(0) \sinh \left(\sqrt{\kappa_{\mathrm{s}} \kappa_{\mathrm{i}}^{*}\left|A_{\mathrm{p}}\right|^{2} \mathrm{z}}\right)
\end{array}\right.
$$

Writing down the complex field amplitudes using their phase and modulus parts $A_{m}(z)=a_{m}(z) e^{j \varphi_{m}(z)}$, one can verify that the signal field $A_{s}(z)$ retains its initial phase and is simply amplified by the interaction, while the generated idler wave $A_{i}(z)$ has a phase that depends both on that of the pump wave and on that of the signal wave. Noticing that $\kappa_{\mathrm{i}}=\left|\kappa_{\mathrm{i}}\right| e^{j \pi / 2}$, we obtain the following relation $\boldsymbol{\varphi}_{\mathbf{i}}=\boldsymbol{\varphi}_{\mathbf{p}}-\boldsymbol{\varphi}_{\mathbf{s}}+\frac{\pi}{2}$ for a bulk crystal with standard birefringent phase matching.

Similarly, in a perfect periodically poled crystal, the relation between phases of the pump, signal and idler waves can be obtained propagating the waves in the crystal domain by domain and step by step, leading do a similar relation:

$$
\varphi_{\mathrm{i}}=\varphi_{\mathrm{p}}-\varphi_{\mathrm{s}}
$$

More generally, for a non perfect periodically poled crystal (for instance not exactly cut at a finite number of coherence lengths), or when phase matching is not perfect, an additional phase offset can appear in this relation, but this offset is fixed so that there's always a $\varphi_{i}=\varphi_{0}+\varphi_{p}-\varphi_{s}$ relation between the 3 coupled waves in a DFG or parametric amplification process.

Consequently, controlling the pump wave phase gives control over the phase offset difference between the signal and idler waves. As we'll detail later on, this relation is enough for allowing indirect phase control of the idler wave with sole control of the pump wave phase. 


\section{EXPERIMENTAL SETUP}

A schematic of the experimental set-up for DFG combining is presented in Figure 1. It's almost identical to the set-up detailed in our previous paper ${ }^{2}$, except for some components such as the mid-infrared detector and the phase control electronics that have slightly evolved since then.

Two 1.06- $\mu \mathrm{m}$ Yb:fiber amplifiers are seeded by a master oscillator (MO), which is a Orion laser module from RIO. These two amplifiers are homemade ytterbium-doped fiber amplifiers, each of them capable of delivering up to $100 \mathrm{~W}$ continuous wave $(\mathrm{cw})$ power. A standard fiber-coupled electro-optic modulator (Photline) controls the phase of one of the $1.06 \mu \mathrm{m}$ fiber amplifiers. This EOM provides both the phase modulation for frequency-tagging and the proper pump wave phase shifts to compensate for the phase fluctuations.

To generate the signal seed at $1553 \mathrm{~nm}$, a laser diode is used as a master oscillator. Amplification of this oscillator is achieved using a commercial erbium-doped fiber amplifier (Keopsys, $10 \mathrm{~W}$ ) split in two to seed both DFG channels with a common signal wavelength.

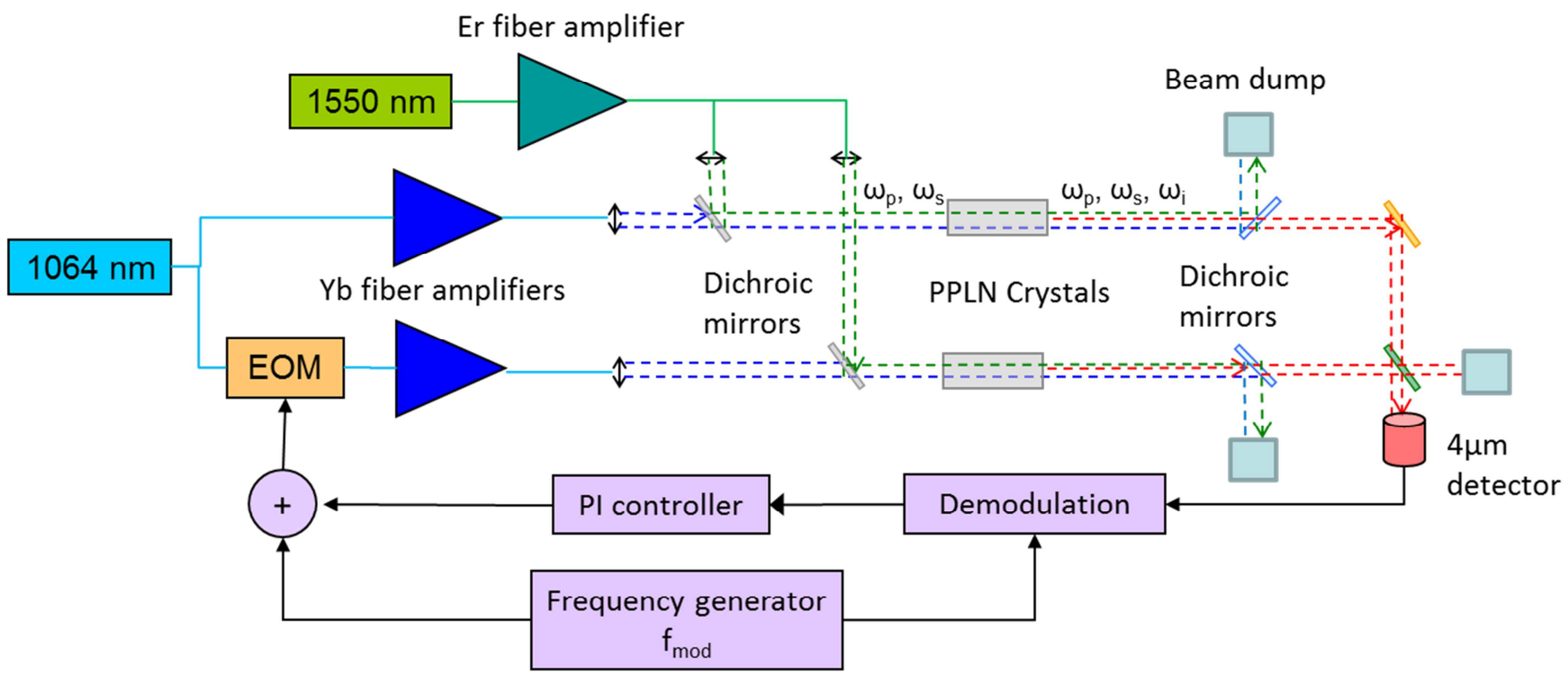

Figure 1. Schematics of the experimental set-up for demonstrating coherent combining of two 3.4- $\mu \mathrm{m}$ idler beams generated through DFG in PPLN crystals. Coherent combining is achieved by active phase-control of one of the pump waves.

Signal and pump beams are collimated to the same diameter and overlapped. Half-wave plates are used to align the linear polarizations of the 4 beams along the z-axis of the PPLN crystal. The two PPLN crystals are from Covesion and their length is $\mathrm{L}=20 \mathrm{~mm}$. The poling period chosen is $30.49 \mu \mathrm{m}$ and the operating temperature is $60{ }^{\circ} \mathrm{C}$ for both crystals, in order to generate an idler wave at $3.4 \mu \mathrm{m}$, corresponding to a signal wave at $1553 \mathrm{~nm}$, the wavelength of the signal seeder. The pump beams are focused down to a waist of $75 \mu \mathrm{m}$ in the PPLN crystals.

At the output of each nonlinear crystal, a dichroic mirror separates the residual signal and pump beams from the 3.4- $\mu \mathrm{m}$ idler beam. The two idler beams are then overlapped using a 50/50 pellicle beam splitter, and the far-field interference signal at $3.4 \mu \mathrm{m}$ provides the feedback necessary to close the phase-locking loop. A coated germanium window filters off the residual signal and pump photons, so that the infrared detector used for phase control only receives the $3.4 \mu \mathrm{m}$ beams interference pattern. This photodetector is an InSb cooled detector.

Frequency tagging operates at $56 \mathrm{kHz}$, and phase error signal generation is performed using a commercial lock-in amplifier. This error signal is driven to 0 by a proportional integral (PI) controller, used to send the proper command in terms of pump wave phase shift on the EOM. 


\section{PHASE CONTROL AND PHASE MEASUREMENT EXPERIMENTS}

\subsection{Phase measurement and indirect phase control tests}

Before performing indirect phase control, we first verified the phase relation between the pump, signal and idler waves. To measure phase differences, the pairs of pump, signal and idler beams are overlapped to create an interference pattern at each of the three coupled wavelengths. An IQ detection is implemented for each of the pump, signal and idler wavelengths.

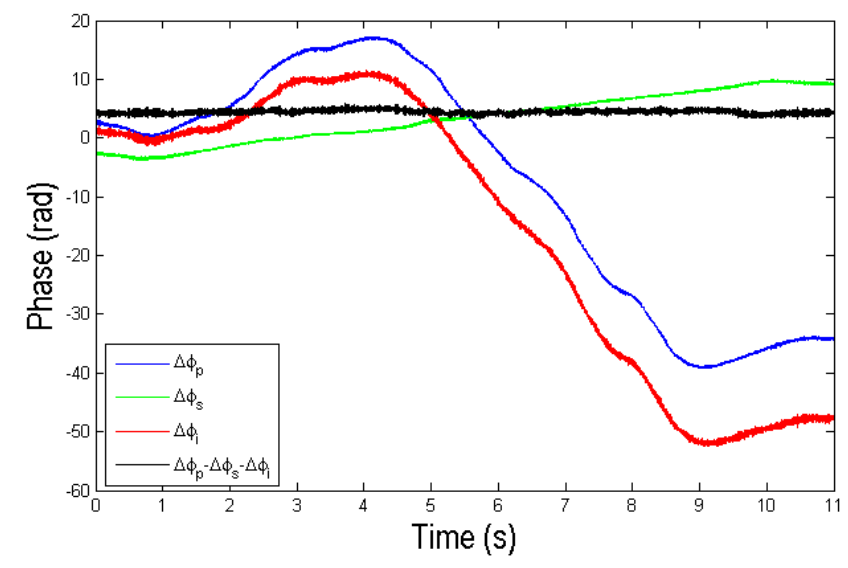

Figure 2. Phase measurements on the 3 coupled waves, when phase control loop is open (i.e. phase control is inactive). The phase relation between the 3 waves is confirmed when they are freely fluctuating in phase.

Fig. 2 presents an example of simultaneous free phase fluctuation measurements for the 3 coupled waves. We measured the phase difference between each pair of waves at the output of the two nonlinear crystals $\Delta \varphi=\varphi_{2}-\varphi_{1}$ for the pump, signal and idler. The difference $\Delta \varphi_{p}-\Delta \varphi_{s}-\Delta \varphi_{i}$ is also plotted. According to equation (4), this difference should be equal to zero. These measurements confirm the $\varphi_{i}=\varphi_{0}+\varphi_{p}-\varphi_{s}$ relation mentioned before, corresponding to the case of a non-perfect periodically poled crystal.

This relation also confirms that indirect phase control should operate properly. But as DFG is a 3-wavelength nonlinear process, one could expect the need to actively control simultaneously the phases of two of the coupled waves to achieve efficient CBC. In practice, it is not the case as active phase control of the sole pump wave is sufficient to efficiently combine the idler waves. The reason for that is explained by the fact that the seeding by the 1553-nm Er:fiber amplifier of both signal waves results in a fixed phase difference between the two signal waves. More detailed explanation have been provided on this matter in our previous paper ${ }^{2}$.

Consequently, a phase shift induced by the EOM on pump wave \#2 will result directly in an effective equal phase shift on idler wave \#2. There's no need to control the signal wave phase and sole control of the pump wave is sufficient for indirect phase control to operate as expected.

\subsection{Coherent combining of the idler waves by indirect phase control}

We verified experimentally this fact and demonstrated the feasibility of DFG idler waves coherent combining with sole control of the pump wave phase.

Fig. 3 presents the time evolution of the interference signal between the two idler beams when driving the pump beam phase with the EOM. 


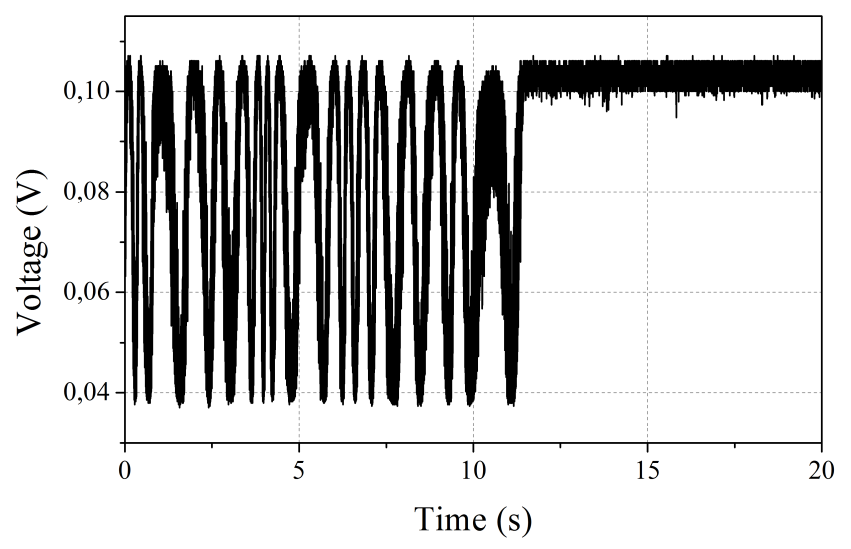

Figure 3. Time evolution of the interference signals of the idler beams when the phase control loop is open and then closed.

When the phase control loop is open and coherent combining inactive, this interference signal fluctuates very fast between a destructive state and a constructive state of interference. As when the loop is closed and the combining becomes active, the interference signal is locked on the constructive interference state.

A residual phase error of $\lambda / 28 \mathrm{rms}$ for the idler wave is measured using IQ detection. It corresponds to an excellent efficiency for coherent combining, demonstrating experimentally the feasibility of DFG coherent combining by indirect active phase control.

\subsection{Limitations of coherent combining in a 3-wavelength nonlinear process}

However, there is a huge difference between the capability of SHG coherent combining and DFG coherent combining. This difference is due to the fact that SHG is a degenerate process effectively coupling two wavelengths, the fundamental wave and the second harmonic wave, and DFG is a process coupling three different wavelengths.

We demonstrated that in some cases, it was possible to perform CBC simultaneously for the fundamental and harmonic beams in the case of SHG CBC.

Here, in the case of DFG CBC, such simultaneous combining is not feasible anymore as Fig. 4 demonstrates. In this figure, IQ detection is applied to pump waves and signal waves while idler waves are coherently combined. It shows that the pump waves and the signal waves are not in phase.

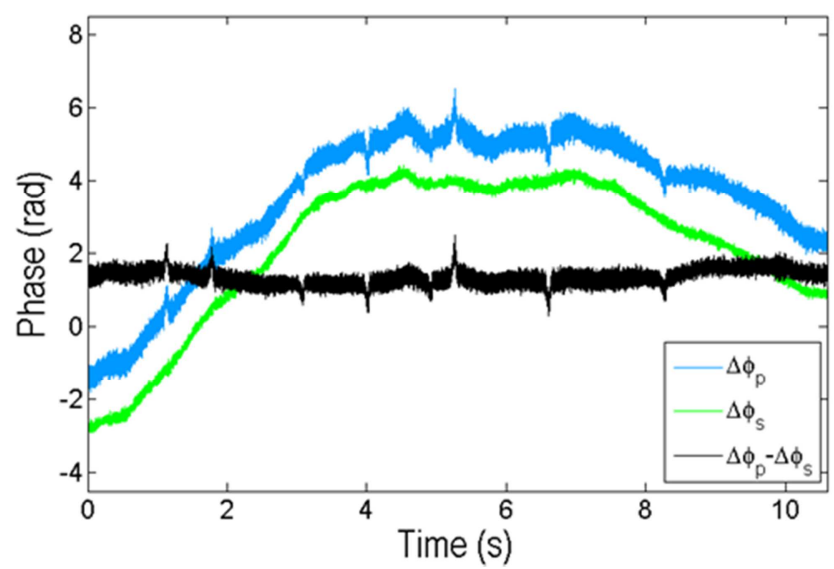

Figure 4. Measurement of the phase difference between the pump and signal waves when the phase control loop on the idler waves is closed. 
Phase control is locked on the idler wave interference signal, and combining is efficient for those idler waves only. The pump waves are phase shifted so that the idler waves are kept in phase but there's no reason for these pump waves, or for the signal waves whatsoever, to be in phase at the same time.

In Fig. 4, as CBC is performing adequately on the idler waves, we know that those waves are in phase. However, the phase difference between the pump waves and between the signal waves still fluctuate freely in this case, due to slow temperature variations inducing uncompensated for slow evolution of the optical path differences for these waves.

\section{COHERENT COMBINING OF OPO}

This experiment proving that control of the sole pump wave phase was enough to efficiently combine the idler waves was a needed intermediate step towards trying to combine OPOs. First, we wanted to be sure that this theoretically feasible phase control was experimentally verified.

Now that we're sure of it, we are considering combining mid-infrared OPOs the same way we did for DFG modules. Basically, the experiment is exactly the same as the one on Fig. 1, except the nonlinear crystal are now inside an OPO cavity and there's no seeding needed.

Another difference is that there's no need anymore for the 1553-nm seed used in DFG to make sure both signal waves are operating at the same wavelength. In the case of an OPO, such wavelength control can now be done by tuning the cavity length, and should be feasible without any seeding of the signal waves, considerably simplifying the CBC configuration and compensating somehow for the added complexity of dealing with the OPO cavities.
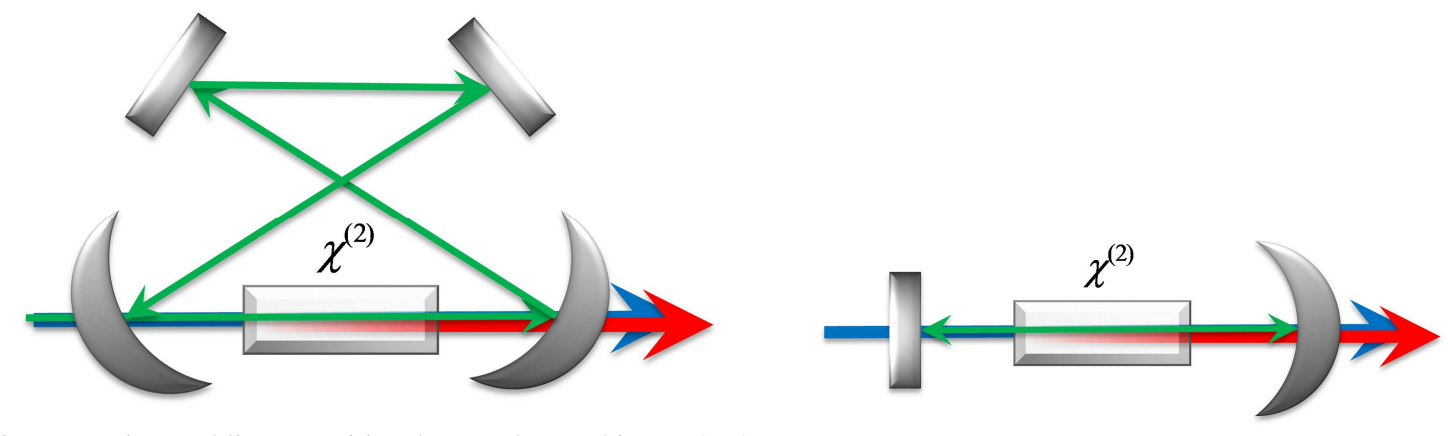

Figure 5. Ring and linear cavities that can be used in cw OPOs.

OPOs can operate in linear or ring cavities (see Fig. 5). The first experiments will use a ring cavity, as it offers the lowest threshold of operation and the highest nonlinear efficiency.

One of the advantages of trying to combine OPOs is a strong increase in the efficiency of the nonlinear process. When the power efficiency of the DFG process was extremely low (around $0.5 \%$ but it wasn't the point of the CBC experiments to break records in terms of efficiency), cw OPOs can reach $10 \%$ efficiency or even more, making it easier to obtain a strong interference signal to close the phase control loop.

At the moment, the OPOs are being tested individually and the CBC experiments will take place soon.

\section{CONCLUSION}

Efficient combining of a pair of difference frequency generators emitting at $3.4 \mu \mathrm{m}$ has been achieved with a residual phase error lower than $\lambda / 28 \mathrm{rms}$ in this DFG CBC experiment.

For the DFG three-wavelength process, we were able to phase lock the idler beams using only one control loop driving the pump wave phase. However, simultaneous combining of the pump waves or of the signal waves was demonstrated impossible to achieve.

Future work is dedicated to applying the same indirect phase control technique to the coherent combining of continuous wave OPOs. 


\section{REFERENCES}

[1] Odier, A., Durécu, A., Melkonian, J. M., Lombard, L., Lefebvre, M., and Bourdon, P., "Coherent combining of second-harmonic generators by active phase control of the fundamental waves," Opt. Lett. 42(16), 3201-3204 (2017).

[2] Odier, A., Durécu, A., Melkonian, J. M., Lombard, L., Lefebvre, M., and Bourdon, P., "Coherent combining of fiber-laser-pumped $3.4 \mu \mathrm{m}$ frequency converters," Proc. SPIE 10083, 1008319 (2017). 